CHERCHER, REPÉRER, AVANCER

\title{
TIRAGE AU SORT ET ASSOCIATIONS ÉTUDIANTES : UNE EXPÉRIENCE DÉMOCRATIQUE À L'UNIVERSITÉ DE LAUSANNE ?
}

Maxime Mellina

De Boeck Supérieur | « Participations »

2019/1 N²3 | pages 147 à 170

ISSN 2034-7650

ISBN 9782807392908

Article disponible en ligne à l'adresse :

https://www.cairn.info/revue-participations-2019-1-page-147.htm

Distribution électronique Cairn.info pour De Boeck Supérieur.

(C) De Boeck Supérieur. Tous droits réservés pour tous pays.

La reproduction ou représentation de cet article, notamment par photocopie, n'est autorisée que dans les limites des conditions générales d'utilisation du site ou, le cas échéant, des conditions générales de la licence souscrite par votre établissement. Toute autre reproduction ou représentation, en tout ou partie, sous quelque forme et de quelque manière que ce soit, est interdite sauf accord préalable et écrit de l'éditeur, en dehors des cas prévus par la législation en vigueur en France. Il est précisé que son stockage dans une base de données est également interdit. 


\title{
Tirage au sort et associations étudiantes : une expérience démocratique à l'Université de Lausanne?
}

\author{
> Maxime Mellina
}

\section{> Résumé}

La Fédération des associations d'étudiant·e.s, syndicat étudiant de l'Université de Lausanne, introduit en 2013 le tirage au sort comme mode de désignation des représentants de son assemblée législative. Avant cette introduction, toutes les personnes immatriculées à l'université votaient pour désigner les étudiants qui les représenteraient au cours de l'année académique. Cet article analyse les dynamiques politiques de cette expérience particulière afin d'amener au débat sur le tirage au sort un éclairage utile dans le questionnement relatif aux pratiques démocratiques. Cette question implique de s'interroger sur les profils des représentants, en montrant que le sort diversifie bel et bien ceux-ci dans le sens d'une meilleure représentativité. Nous revenons ensuite sur les effets symboliques de la sélection aléatoire, qui conduisent dans notre cas à la démystification du rôle de représentant et à une plus grande prise en considération de l'intérêt de la communauté dans la délibération. Nous discutons enfin comment la dimension représentative et la dimension symbolique se réalisent dans le processus dynamique de la délibération. Cette analyse nous permet de mettre en évidence que bien que le tirage au sort réduise la force des structures génératrices de rôles, il ne semble pas ici engager d'effets assez puissants pour déstructurer totalement les normes et les codes classiques du jeu politique. 
«Ce qui est distribué de façon égale par le sort n’est pas le pouvoir, mais la probabilité, au sens mathématique, d'accéder au pouvoir » (Manin, 2012 [1995], p. 58).

Après une année d'essai, la Fédération des associations d'étudiant.e.s (FAE) de l'Université de Lausanne introduit le tirage au sort comme mode de désignation des représentants de son assemblée législative 1 . Depuis 2013, les statuts de l'association consacrent un tirage au sort en deux temps: une première sélection est effectuée parmi les 14000 étudiants de l'université pour désigner les 23 représentants qui siègent dans l'assemblée. Lors de ce premier tour, le centre informatique de l'université sélectionne 23 étudiants et transmet les noms au secrétariat de la FAE qui a pour mission de les contacter. Ceux-ci, avertis par e-mail, peuvent refuser leur élection, ce qui arrive régulièrement : en effet, entre deux et cinq étudiants seulement acceptent chaque année cette élection et il est même arrivé une année qu'aucun étudiant n'accepte cette désignation du premier tour. Un second tirage est alors effectué parmi une liste d'environ 40 volontaires, pour compléter les sièges laissés vacants par les refus ou les non-réponses des 23 premiers sélectionnés. Avant cette introduction, toutes les personnes immatriculées à l'université pouvaient voter selon un scrutin proportionnel pour des listes partisanes et désigner les étudiants qui les représenteraient au cours de l'année académique?

Cette nouvelle pratique est digne d'intérêt puisqu'il est particulièrement rare de pouvoir observer les dynamiques politiques propres à la sélection aléatoire dans une structure permanente et réglée. En effet, peu d'exemples contemporains tirent au sort des représentants directement liés à un espace de prise de décision et où ceux-ci débattent autour de nombreux sujets : il existe par exemple les assemblées constitutionnelles estonienne et irlandaise (Suiter, Farrell, Harris, O'Malley, 2019) et le Conseil supérieur de la fonction militaire français (Courant, 2019). À la FAE, ces représentants composent le «parlement» de l'institution dont les structures rappellent le dispositif classique de séparation des pouvoirs propre aux gouvernements représentatifs - selon les mots de Bernard Manin (2012 [1995]) - divisé entre une assemblée législative, un collège exécutif et une administration. L'Assemblée des délégué·e-s est composée de 23 membres élus (puis tirés au sort dès 2012), qui représentent la communauté universitaire et 23 membres qui représentent les associations facultaires autonomes. Celles-ci sélectionnent leurs représentants selon des logiques différentes (cooptation, élection, désignation). L'assemblée se réunit une fois par mois et décide des

[1] Cet article est publié avec le soutien du Fonds national suisse de la recherche scientifique (projet «Expériences du tirage au sort en Suisse » n 163126 et FNS Scientific Exchange n¹0C017_175429). Il s'agit d'un développement d'une recherche effectuée dans le cadre d'un travail de master à l'Université de Lausanne (Mellina, 2016).

[2] Les informations factuelles sont fondées sur les statuts et les procès-verbaux de l'association du 29 septembre 2009 au 28 avril 2015, ainsi que sur des entretiens avec le secrétaire général et le coprésident de l'association. 
lignes politiques de l'association. Le bureau exécutif compte huit membres élus par l'assemblée législative et s'occupe, avec le secrétaire général employé à $90 \%$, de la gestion interne de l'association et de la représentation des étudiants auprès des instances académiques et politiques.

La spécificité des dynamiques de représentation propre au monde associatif et universitaire double aussi l'intérêt de ce cas. En effet, «la représentation des étudiants est un objet privilégié pour examiner les relations diverses qui permettent à des acteurs individuels ou collectifs de parler et d'agir au nom d'un groupe » (Le Mazier, Testi, Vila, 2014, p. 214 ; voir aussi Le Bart, Merle, 1997). Toutefois, il ne faut pas non plus oublier que bien que la FAE reste une association non professionnelle, elle s'inscrit dans un sous-champ politique et académique qui nécessite une attention quotidienne de ses membres. La FAE est une association reconnue par les autorités universitaires et cantonales qui la considèrent comme l'unique partenaire privilégié et représentatif de l'avis des étudiantes et étudiants. Elle est, sur la base de ses statuts, un véritable syndicat défendant l'intérêt de la communauté estudiantine. Les membres du bureau exécutif rencontrent la direction de l'université et le ministre cantonal en charge de la formation une fois par semestre, et tentent d'influencer les autorités autour de nombreux sujets (la hauteur du montant des taxes d'études, les bourses aux étudiants ou les logements pour ceux-cil. L'association est un acteur qui compte et qui a obtenu plusieurs succès ces dernières années, notamment au sujet des bourses d'études ou de la politique du logement. Les étudiants tirés au sort à la FAE disposent donc d'un véritable poids décisionnel au sein des structures de l'association et définissent, en tant que représentants de l'assemblée législative, les lignes politiques directrices de celle-ci.

Cette proposition est également digne d'intérêt puisqu'elle s'inscrit, dans les discours des acteurs, dans la crise des institutions politiques représentatives des démocraties contemporaines (Blondiaux, 2008 ; Sintomer, 2011a, p. 15-38). Cette crise s'accompagne d'une importante réactivation de l'intérêt que l'on porte à la pratique du tirage au sort dans l'optique d'une critique des conceptions élitistes de la représentation (Rancière, 2005 ; Stone, 2009, p. 375-377). À la FAE, les acteurs eux-mêmes pensent leur démarche comme une proposition politique radicale : le sort y est défendu par un petit groupe d'étudiants en science politique pour qui le tirage au sort garantit une égalité face à la sélection, en réglant en partie les problèmes classiques de participation (fort taux d'abstention aux élections et faible implication des représentants étudiants élus), en abaissant les barrières d'entrée à l'assemblée et en supprimant les conflits partisans devenus stériles ${ }^{3}$. Le retour du tirage au sort, à la FAE comme ailleurs, a donc pour conséquence l'ouverture d'un champ politique et scientifique qui expose les logiques essentiellement distinctives des systèmes représentatifs

[3] La genèse et les raisons de l'instauration de cette nouvelle procédure ne constituent pas l'objet de cet article. Elles sont analysées dans Mellina (2016, p. 28-46). 
occidentaux (Bourdieu, 1981 ; Dahl, 1989), contraires à la nécessité égalitaire des systèmes délibératifs démocratiques (Barber, 1997 [1984] ; Talpin, 2019). En ce sens, le cas diffère des premières propositions contemporaines de réintroduction du sort en politique qui ont vu le jour dès les années 1970 (Bacqué, Sintomer, 2010 ; Garibay, 2015), et qui étaient liées à l'idée de la démocratie délibérative et à l'utilisation du sort au sein d'institutions consultatives ljurys citoyens, conférences de consensus). Comme le montre Yves Sintomer (2019), l'expérience de la FAE fait partie d'une nouvelle vague d'expériences qui se revendique d'un courant plus radical qui souhaite intégrer le hasard dans des formes créatives et directement liées à des espaces de prise de décision.

Cet article analysera donc les dynamiques propres au tirage au sort en tant qu'outil de délégation du pouvoir dans une instance décisionnelle permanente. Il ne s'agira pas d'analyser les conditions sociales de la constitution des qualifications politiques et techniques que demande la participation politique, ni même de brosser un profil social et politique exhaustif des représentants ou encore de l'ensemble des étudiants, comme a pu le faire Sébastien Michon par exemple (2008). Il faudra plutôt observer si la FAE connaît aussi les mécanismes de concentration du pouvoir par un petit nombre d'individus et si le tirage au sort, par les effets politiques qu'il déploie, permet de limiter le champ de forces qui facilite cette concentration des fonctions. Il faudra discuter les éventuels effets égalitaires du sort dans ce contexte, en se demandant s'il permet de diversifier le profil politique des représentants et d'endiguer les logiques partisanes. Pour Daniel Gaxie (1993 [1978]), la politisation varie en fonction de la compétence à évoluer et donner un sens au champ politique. C'est pourquoi j'ai particulièrement considéré la compétence politique des représentants, c'est-à-dire leur compréhension fine du jeu politique, des normes de la délibération et des sujets traités. Il faudra également se demander si le tirage au sort permet de neutraliser la compétition politique - en étant le produit d'effets psychologiques, symboliques et collectifs - et s'il a des effets in fine sur le processus délibératif démocratique. Ces questionnements sont d'ailleurs renforcés dans ce cas par la possibilité d'une comparaison entre des représentants élus (avant 2012) et des représentants tirés au sort, opposition déjà discutée par la théorie politique (Malkopoulou, 2014).

Ces analyses se fondent sur une enquête de terrain menée à l'Université de Lausanne. Elle comprend l'examen du matériel produit par l'association (procès-verbaux, liste des représentants, site Internet), des discours produits par ses membres (comme dans le journal des étudiants, L'auditoire) et sur cinq années d'observation participante (de la rentrée académique 2010 à la fin de l'année 2015). Durant cette période, de nombreux discours ont également été récoltés par l'intermédiaire de 69 questionnaires auprès des délégués élus puis tirés au sort entre 2010 et 2015 (44\% de réponses), et complétés par neuf entretiens qualitatifs. L'observation participante s'est terminée en 2015, date marquant la fin de la recherche. Toutefois, la FAE continue d'utiliser cette procédure jusqu'à aujourd'hui selon les mêmes modalités. 
Il s'agira donc tout d'abord de décrire les effets de cette nouvelle norme sur les profils des étudiants qui participent à l'assemblée. Une première partie traitera de l'accès égalitaire à la délibération politique à travers le prisme de la représentativité. Une seconde partie reviendra sur les effets symboliques et collectifs du sort, ressentis par les représentants. Enfin, une dernière partie se focalisera sur le lieu de la délibération et les effets du sort sur le processus politique délibératif dans l'assemblée.

\section{Vers plus de représentativité ?}

L'augmentation de la participation politique des étudiants est l'un des buts les plus importants de l'instauration du tirage au sort à la FAE. Ses promoteurs condamnaient une vision de la démocratie dans laquelle la professionnalisation de la politique et le monopole des débats par des listes partisanes rendaient trop difficile la participation de 《profanes $»^{4}$. Ce désintérêt de certains groupes d'étudiants pour les actions de la FAE était cristallisé par la faible participation aux élections (entre $5 \%$ et $9 \%$ de participation avant 2012), par l'impossibilité de réunir 150 étudiants pour pouvoir convoquer une assemblée générale, et par la difficulté de trouver des représentants motivés pour les instances de l'université. Le tirage au sort devait alors stimuler la participation d'étudiants lambda dans les instances décisionnelles de l'association, du fait notamment que son mécanisme permet d'améliorer la représentativité sociologique des délégués en fonction de critères (comme l'âge, le sexe ou le degré de politisation) et indépendamment des logiques partisanes ${ }^{5}$. Concrètement, quels étaient les profils des étudiants qui constituaient les représentants avant l'instauration du tirage au sort ? Et quels changements peut-on observer après l'instauration du tirage au sort?

\section{Le profil des représentants : du partisan à l'intéressé}

Avant l'instauration du tirage au sort, le secrétariat général de l'association était chargé d'organiser les élections au début de chaque année académique, et tous les étudiants de l'université étaient appelés à voter pour des listes - représentant des orientations politiques. Chaque étudiant recevait un courriel (puis deux ou trois rappels) dans lequel on lui donnait la possibilité de voter électroniquement. De leur côté, les candidats devaient se regrouper et former des listes pour obtenir des suffrages. Dans ce cadre, le profil des élus se rapproche de celui des professionnels de la politique. Les délégués élus sont très politisés

[4] FAE, Procès-verbaux de l'Assemblée des délégué.e.s du 23/05/2012 et du 14/02/2013. Entretiens avec le secrétaire général, décembre 2012 et avec le coprésident de l'association, octobre 2015.

[5] «La FAE opte pour le tirage au sort », par Étienne Kocher, dans L'auditoire, octobre 2012, p. 12. 
(Gaxie, 1993 [1978] ; Lagroye, 2003), c'est-à-dire qu'ils savent évoluer dans ce champ politique. Ils connaissent à la fois les sujets traités, mais aussi les codes du débat et de la négociation politique. Comme le rappelle Loïc Blondiaux (2001, p. 48), « ce sont toujours plutôt les individus les mieux intégrés politiquement, les mieux formés et les plus au courant des codes politiques qui font vivre les institutions, y investissent leur énergie et y exercent leur compétence ». La FAE n'échappe pas à cette règle.

La connaissance des codes politiques s'explique en partie par les liens étroits qui existent entre les partis politiques et les listes électorales sur lesquelles les étudiants s'inscrivent pour participer aux élections et accéder à l'assemblée. Il existe trois groupes politiques: le centre droit (Centre Droit Universitaire), la gauche sociale-démocrate (la Gauche étudiante) et la gauche radicale (Alternative étudiante). Ces listes sont fortement affiliées aux principales jeunesses des partis de la politique vaudoise, les jeunes libéraux-radicaux, les jeunes socialistes et les jeunes verts. Comme dans d'autres exemples d'associations étudiantes (Le Mazier et al., 2014), les élections rendent la représentation particulièrement concurrentielle à la FAE. Or, cette configuration partisane limite la diversité des profils dans l'assemblée, puisque les membres des listes recrutent largement la relève au sein de leurs affiliations partisanes respectives. Ainsi, plus de la moitié des délégués élus étaient membres d'un parti avant leur arrivée à la FAE. Les représentants étaient donc à la fois des individus socialisés aux codes politiques en partie par l'intermédiaire de leurs expériences partisanes et des représentants qui bénéficiaient d'une connaissance fine des dossiers en discutant en groupe des thématiques avant l'assemblée.

Pour les élus, la FAE constitue une étape d'un cursus honorum spécifique à la professionnalisation de la vie politique (Gaxie, 1997). Ceux-ci consacrent une part importante de leur vie à la politique et concentrent «l'essentiel de leurs intérêts sociaux sur cette activité particulière » (p. 28). Sauf échec personnel, celles et ceux qui entrent dans l'univers de l'activité politique tendent donc à y rester. On liste d'ailleurs lors d'un entretien le nombre non négligeable de politiciens passés par la FAE (dont plusieurs conseillers nationaux et un conseiller d’État vaudois).

L'analyse des profils des représentants tirés au sort montre une évolution intéressante. Il faut noter d'abord que les profils des représentants ne se transforment pas radicalement, puisque les délégués tirés au sort, ceux qui acceptent leur élection au premier tour ou les volontaires, gardent tous un rapport formé à la « chose politique » dans son acception large lon trouve par exemple le cofondateur d'un journal d'opinion ou une militante féministe). Ce qui change, ce n'est pas leur intérêt propre pour le politique, mais leur plus faible connaissance et compréhension du jeu politique, de ses codes, et de la maîtrise d'un langage spécifique, qui est l'apanage de professionnels. Plusieurs discours de ce type montrent cette évolution : 
« Je ne savais pas tellement ce que c'était qu'un PV ou un ordre du jour avant, mais [...] on avait demandé d'expliquer un peu tout ça et on avait reçu un vade-mecum fait par le bureau. [...] Bon et le bureau de toute façon il explique les abréviations quand on ne sait pas trop ce que c'est » (entretien Justin'b).

De plus, j'ai demandé systématiquement aux délégués élus puis tirés au sort s'ils avaient d'autres engagements politiques à côté de la FAE. Leurs réponses illustrent clairement cette évolution: on ne trouve plus que trois représentants qui mentionnent leur appartenance à un parti entre 2012 et 2015 et plus on avance dans le temps, plus ces profils tendent à disparaître. Les délégués tirés au sort inscrivent alors plutôt leurs engagements au sein de l'université, comme dans le cadre d'autres associations facultaires ou d'autres associations estudiantines.

La majorité des délégués tirés au sort conservent leur capacité de verbaliser de façon construite, mais peu partisane, des arguments et des positions politiques. Cet état de fait s'explique sans doute parce que les étudiants sélectionnés par le sort peuvent refuser leur élection et qu'un deuxième tirage est effectué sur un panel de volontaires. Dans ce contexte, la sélection distingue toujours les plus concernés et les plus disponibles. L'expérience montre en tout cas que l'introduction du tirage au sort - ou du moins, la suppression des listes politisées - a pour effet de supprimer le lien presque institutionnalisé entre certains partis et l'association et par là même diminue le profil très partisan des représentants. Le tirage au sort diversifie donc bien les profils et fait apparaître quelques profils atypiques, notamment au regard de la division entre initiés et profanes (surtout pour les tirés au sort du premier tour).

\section{Représentativité}

En termes de représentativité descriptive, l'analyse de la participation des femmes constitue un indicateur intéressant ${ }^{7}$. Les femmes élues lavant 2012) sont alors sous-représentées au regard du nombre d'étudiantes dans leur ensemble : parmi les élus des années 2010-2011 et 2011-2012 que j’ai pris en considération dans mon étude, je compte 40 individus $^{8}$ dont seulement 10 sont des femmes, ne représentant que $25 \%$ des individus; parmi les tirés au sort des années 2012-2013, 2013-2014 et 2014-2015, je compte 64 individus ${ }^{9}$ dont

[6] Les noms ont été anonymisés.

[7] La proportion de femmes était de $55 \%$ en 2014 à l'UNIL, tous niveaux d'études confondus.

[8] Et non pas 46 individus, c'est-à-dire deux années x 23 sièges, notamment car une part des délégués se représente d’année en année.

[9] Trois tirages de 23 personnes avec quelques délégués qui se sont représentés et ont été à nouveau tirés au sort ( 5 personnes). 
25 femmes, représentant $39 \%$ des élus. Dans cette deuxième période, on remarque que le tirage au sort a bien diminué les mécanismes genrés qui se déploient de manière plus générale dans l'accès aux fonctions dirigeantes.

Un autre indicateur intéressant est la provenance facultaire des représentants. En effet, comme l'a montré S. Michon (2008), si la politisation des étudiants s'explique pour partie par leur origine sociale et leur socialisation à la politique au sein de leur famille, l'action du contexte d'études ne doit pas être rejetée. Il montre que la politisation est plus élevée dans les filières de sciences humaines et sociales que de sciences et techniques parce que les premières favorisent l'activation et l'acquisition de dispositions à la politisation. Dans notre cas, avant l'instauration du tirage au sort, le taux de représentants provenant des sciences humaines est caractéristique de ces résultats, puisque $60 \%$ des représentants étaient issus de la Faculté des sciences sociales et politiques et de la Faculté des lettres ${ }^{10}$. À l'inverse, aucun des représentants n'est issu de la Faculté de biologie et médecine. Après l'instauration du tirage au sort, cette proportion diminue quelque peu : $46 \%$ des représentants proviennent des facultés des sciences humaines et on trouve alors deux représentants de la Faculté de biologie et médecine.

La sélection aléatoire permet donc dans une certaine mesure ce que Samuel Hayat (2013) appelle la «représentation inclusive», c'est-à-dire une plus grande inclusion des individus avec une plus faible considération de leur identité sociale. On l'a vu, la composition des représentants tirés au sort fait apparaître que les participants ne sont plus ni des professionnels ni, pour une partie d'entre eux au moins, des «associatifs », mais qu'ils ne sont pas pour autant des étudiants quelconques, au sens de représentatifs sociologiquement: très peu d'étudiants acceptent leur sélection lors du premier tirage parmi l'ensemble des étudiants lentre 2013 et 2015, 2 à 4 individus acceptaient de siéger et cette proportion reste la même jusqu'à aujourd'huil. Cette conclusion est partagée par l'analyse empirique des jurys citoyens à Berlin de Anja Röcke (2006, p. 27) : «Le tirage au sort n'est pas une procédure miracle qui produit automatiquement un "microcosme" de la population ». Jean-Michel Fourniau (2019) montre également que dans les dispositifs reposant sur le volontariat, le mélange entre hasard, disponibilités et motivations des participants diminue la représentativité du sort.

Néanmoins, cette nouvelle procédure parvient tout de même à limiter les écarts de représentation qu'on observait avant 2012 avec le mécanisme de l'élection, notamment en permettant deux libertés clefs que les délégués mettent en

[10] Alors que ces deux facultés ne représentent que $45 \%$ de l'ensemble des étudiants immatriculés en 2017. Voir UNIL (Université de Lausanne), 2018, Statistiques en ligne, Lausanne, UNISIS, https://www.unil.ch/statistiques/home/menuinst/statistiques-en-ligne.html laccès le 05/03/2019). 
avant dans leurs discours : d'une part, la suppression de l'obligation de choisir un parti en raison de la suppression des listes électorales qui rendaient réelle l'instauration d'une élite. D'autre part, la suppression de la nécessité de faire campagne pour être élu, ce qui réduit l'impératif de posséder un capital politique élevé pour se présenter - bien savoir parler, pouvoir réaliser des flyers, etc. La neutralisation des procédures que permet le tirage au sort a réussi à la FAE à supprimer l'idée d'une mise en avant et d'une compétition aux niveaux personnels et partisans.

\section{Le système résiste}

Le tirage au sort n'est toutefois égalitaire que dans son mécanisme (Delannoi, $2011 b$, p. 155), c'est-à-dire que son utilisation est très flexible selon son intégration dans une procédure et selon la souplesse que permet son application. $\grave{A}$ la $F A E$, on constate certes une multiplication des profils grâce au mécanisme de la sélection aléatoire, mais il est aussi possible d'observer que le «système résiste » à cette rotation forcée par au moins trois moyens.

D’abord, par la possibilité d'une réinscription au tirage de l'année suivante : la faible participation de l'ensemble du corps estudiantin à ce tirage offrant une probabilité assez haute d'être à nouveau sélectionné. En effet, il n'y a que cinq étudiants au mieux qui acceptent leur élection au premier tour et entre 30 et 40 étudiants qui s'inscrivent chaque année au tirage du deuxième tour. La proportion des volontaires au regard de l'ensemble des 14000 étudiants de l'université reste donc très faible.

Deuxièmement, par la possibilité de continuer de siéger au sein de l'assemblée en tant que représentant des associations facultaires.

Enfin, par la possibilité d'un transfert vers le bureau exécutif de l'association, consacrant ainsi ce qu'on peut appeler la progression classique d'une carrière politique estudiantine. L'application concrète du tirage au sort à la FAE assure donc tout de même qu'un jour ou l'autre, ceux qui étaient volontaires parviennent à exercer la fonction à laquelle ils se sont portés candidats.

De plus, en focalisant l'analyse sur les représentants, il est difficile d'avoir une vision précise d'éventuels effets excluants du sort. Puisque tout fonctionne par tirage au sort, principalement parmi les volontaires, l'immense majorité des étudiants se trouve dès lors exclue de toute forme de démocratie universitaire. Ceux-ci peuvent certes librement s'inscrire au tirage, mais la proportion d'étudiants qui s'y inscrit reste bien plus faible que la proportion de votants avant 2012, et ce malgré un taux de participation extrêmement faible : $5 \%$ des étudiants participaient alors aux élections, ce qui représentait tout de même plus de 700 individus chaque année. En même temps, ce qui fait la représentation en un sens inclusif selon S. Hayat (2013, p. 134), « ce n'est pas que le représentant [...] 
ressemble aux représentés ou défende leur intérêt; c'est que les représentés apparaissent directement sur la scène publique, qu'ils portent des jugements, expriment leurs volontés, contestent ce qui est dit et fait en leur nom, construisent des institutions alternatives ». En cela, le tirage au sort multiplie les possibilités d'intervenir directement, sans toutefois parvenir à mettre totalement en échec dans ce contexte les logiques exclusives de l'élection.

\section{Les effets symboliques de la sélection aléatoire}

Une analyse des effets du tirage au sort ne peut se limiter à la vision formaliste et descriptive de la représentation. Elle doit interroger le caractère substantiel de celle-ci. Pour ce faire, l'analyse de Hanna Pitkin (1972 [1967]), et plus généralement les théories anglo-saxonnes de la représentation, ne suffisent pas à saisir les contraintes symboliques des relations de représentation. La tradition allemande (Göhler, Pégny, Sintomer, 2013) permet de thématiser la représentation symbolique et les effets de l'introduction du tirage au sort sur la « façon de se représenter ce qui est représenté » (Hayat, Sintomer, 2013, p. 10). Dans ce cadre, j'ai donc systématiquement demandé aux délégués élus, puis sélectionnés par le sort, leur perception de la représentation, de leur rôle, leur sentiment de légitimité et leurs relations - concrètes ou imaginées - avec les représentés. Pierre Rosanvallon (2008) discute plus largement des légitimités politiques démocratiques qui ne se réduisent pas aux élections, et Dimitri Courant 12014 , p. $105-110 ; 2018$ ) a conceptualisé quant à lui pour la première fois les « légitimités du sort » en établissant une typologie spécifique de ses trois formes qui ont chacune leurs effets propres: la «légitimité-similarité llégitimes car semblables), la légitimité-horizontalité (légitimes car indépendants) et la légitimité-humilité (légitimes car non supérieurs) », qui reposent respectivement sur ce qu'il qualifie de «principes démocratiques du tirage au sort » : la représentativité, l'impartialité et l'égalité. Je m'inspire de cette approche pour analyser le cas de la FAE.

\section{La légitimité de ressemblance : égalité, démystification et savoir d'usage}

La volonté égalitaire et inclusive fondée sur l'idée d'une amélioration de la représentativité mathématique s'accompagne d'effets symboliques chez les délégués. Cet effet peut être rapproché de ce qu'Hélène Landemore (2010, p. 26) appelle la «légitimité épistémologique » du tirage au sort ou ce que Jane Mansbridge (2013, p. 72) qualifie de «légitimité empirique ». Ainsi, par ce mécanisme de la sélection aléatoire, couplée à une rotation régulière des charges, il est difficile pour les représentants de percevoir une distinction par rapport à celles ou ceux qui n'ont pas été désignés: 
«Quand on m’a réellement appris que j'étais élu [c'est-à-dire tiré au sort], j'y repensais plus. Donc je me suis dit, c'est une deuxième chance qu'on m'a donnée, parce que j'avais complètement oublié. [...] Et [donc], pour moi, franchement, je représente l'étudiant lambda, et l'étudiant lambda, il vote comme les autres. Que ce soit moi ou un autre : pareil ! Je suis de droite, que ce soit moi ou un autre de gauche, c'est pareil. Je représente comme beaucoup pendant l'Assemblée des délégués, un étudiant... normal... qui se retrouve là parce qu'il a été tiré au sort [...] et si je ne suis pas réélu, c'est pas grave, j'en ferai pas une maladie » (entretien Luc).

Le tirage au sort «présuppose alors une conception abstraite des citoyens qui deviennent des unités interchangeables au sein d'une entité élargie » (Röcke, 2006, p. 28). Cette conception a au moins trois effets largement observables.

Le premier effet constaté à la FAE, comme dans d'autres cas, consiste en une inclusion à la communauté et en une égalité symbolique. Comme le représentant n'est plus qu'une unité interchangeable, il a tendance à incarner un tout et il se rattache désormais presque toujours à l'ensemble de la communauté estudiantine. Cette idée est très présente dans les discours des délégués tirés au sort («je représente les étudiants dans leur ensemble » ou encore « je suis un étudiant lambda de l'université »), alors que les délégués élus se rattachaient toujours à leur liste ou aux mandants qui les avaient élus.

Toutefois, une part importante des étudiants sélectionnés par le sort définissent en réalité la « communauté » à laquelle ils se rattachent de façon plus restreinte. La sélection aléatoire complète l'effet communautaire par un effet de proximitép c'est-à-dire que les délégués se sentent in fine plus légitimes à représenter ceux dont ils sont plus proches et auxquels ils ressemblent le plus (les membres de leur faculté par exemple).

Cet effet d'inclusion communautaire et de proximité a un impact sur la production des positions politiques des délégués. On observe en effet un glissement de l'idée d'une légitimité de parti - à la fois au sens d'un parti pris mais aussi au sens d'une organisation fournissant une orientation politique - vers l'idée d'une prise en compte plus large et globale d'une diversité de points de vue. Il faut tout de même noter que cette reconnexion symbolique des représentants n'est pas si claire et homogène dans les discours des délégués et le sentiment de proximité peut être plutôt représenté par un second effet symbolique qui est celui de la rupture entre un savoir savant et un savoir profane. L'introduction de la sélection aléatoire limite considérablement dans l'imaginaire de certains délégués

[11] Pour une discussion critique de la « légitimité de proximité », voir Pierre Rosanvallon (2008, p. 267-344). 
l'opposition traditionnelle entre une opinion éclairée du représentant, élu pour ses «qualités» politiques, et celle d'un étudiant ordinaire. Cette observation va de pair avec l'idée que les représentants se réfèrent de plus en plus à ceux qui leur ressemblent et qu'ils sont donc à même de représenter puisqu'ils les connaissent. Ceux-ci se sentent alors légitimes à donner leur avis pour ceux qui leur ressemblent et font les mêmes expériences qu'eux (dans la même faculté par exemple), mais uniquement sur des sujets qui les concernent directement. Une partie importante des délégués se disent donc être légitimes à prendre position sur la base de leur expérience universitaire ou facultaire, de leur savoir d'usager informé. Il existe donc une rupture entre un savoir savant et un savoir profane (Blondiaux, Sintomer, 2002, p. 31). Cette observation se conjugue avec le fait que les représentants se sentent plus à même de représenter les étudiants qui leur ressemblent le plus ou qu'ils connaissent. Le sort permet donc bien l'intégration de savoirs citoyens (ici étudiants) dans la discussion.

Le troisième effet de la légitimité de ressemblance est celui d'humilité et de démystification du rôle de représentant. D. Courant $(2014$, p. 108 ; 2018) théorise de façon originale ce sentiment d'humilité comme un type de légitimité et affirme : « Le tiré au sort ne s'estime ni meilleur que les autres candidats au tirage ni même supérieur à la majorité de ceux qu'il représente et qui n'ont pas tenté leur chance, tout simplement car il n'y a aucun mérite à avoir été désigné par l'aléa ». Ici, c'est plutôt une démystification du rôle qui joue comme une sorte d'impartialité, transcendant l'idée d'un rattachement à un parti ou à une mouvance idéologique : « employée de façon pure, [la sélection aléatoire] ne fige pas les frontières des groupes et ne réifie pas les identités puisqu'elle n'en présuppose aucune » (Sintomer, 2011a, p. 211).

\section{La légitimité d'indépendance : individualisme et neutralité}

Une autre incidence symbolique de la sélection aléatoire, observée à la FAE comme dans d'autres exemples, est l'indépendance des délégués, «qui ne doivent leur titre à personne » (Courant, 2014, p. 106 ; 2018). Pour une part des représentants, c'est la fonction elle-même, c'est-à-dire le simple fait d'avoir été sélectionné, qui légitime leurs positions. Cette indépendance générée par la sélection aléatoire a un effet neutralisant - bien connu de la littérature sur le tirage au sort - «puisque, contrairement à l'élection, aucune volonté, aucun intérêt ni aucune passion humaine ne sont impliqués dans le processus » (Cervera-Marzal, Dubigeon, 2013, p. 158). Cette observation va de pair, dans notre cas, avec une défiance à l'encontre des intérêts organisés, comme ceux des partis par exemple:

«Tu vois quand tu es dans un parti politique, tu prends tes décisions en fonction d'un programme, on s'aligne sur quelque chose, alors que là j'étais complètement aligné sur moi-même, ce que j'estimais juste, ce que j'estimais pas juste, donc c'était plutôt en fonction de... je ne dirais pas d'une idéologie..., mais plutôt en fonction de... de mes convictions simplement » (entretien Massimo). 
Le tirage au sort limite ici les luttes entre fractions et isole les acteurs de toute idée de groupe, un peu paradoxalement avec l'idée de l'inclusion communautaire développée ci-dessus. L'exemple de Luc est particulièrement intéressant et illustre cet effet neutralisant. Luc se propose sur la liste pour être tiré au sort, il est très jeune, intéressé par la politique, un des rares membres d'un parti lcentre droit) et membre du Conseil communal de son village. Lors d'un entretien, il revient sur ce qui change entre le fait d'être tiré au sort et d'être élu. Il explique sans hésiter que lorsqu'il siège au Conseil communal, il vote presque sans réfléchir comme « le veut son parti », alors qu'à la FAE « être de gauche ou de droite, c'est un peu dérisoire [...]. Ici je prends mes décisions selon mes convictions ». Le sort semble donc neutraliser au sein de l'assemblée de la FAE ses convictions partisanes, et ramène la formation de l'opinion au sein de l'assemblée.

Toutefois, en mettant à distance les positions partisanes et les intérêts particuliers, cette valorisation du citoyen individuel a pour conséquence une dépolitisation relative des débats, puisqu'elle peut tendre «à être désencastrée du social, des relations de force et des cristallisations d'intérêts qui marquent celui-ci » (Sintomer, 2011b, p. 175). Le tirage au sort semble ici aller à l'encontre de l'idée de luttes organisées, et peut parfois susciter une insatisfaction auprès des délégués les plus politisés. Luc ajoute d'ailleurs : « [À la FAE], il n'y a pas de débat contradictoire, c'est ça cette logique de politique politicienne qui manque. Quand il n'y a pas de débat, tu t'ennuies presque » (entretien Luc).

\section{L'(it)légitimité d'infériorité : démotivation, effacement et désincarnation}

Dans les cas analysés par D. Courant (2014, p. 110 ; 2018), « la propre sensation de légitimité-humilité que les tirés au sort [...] éprouvent n'est pas une forme de légitimité-infériorité, il n'y a pas de complexe, de honte ou de sentiment d'infériorité par rapport aux représentants issus des autres procédures de sélection ». À la $F A E$, on note en revanche un sentiment d'infériorité de certains représentants, qui ne comprennent pas pourquoi ou pour qui ils siègent ; ce mécanisme entraîne quelquefois la démotivation, l'effacement ou encore le désengagement.

La sélection aléatoire est parfois présentée, comme le montre Philippe Ségur (2013, p. 688), « comme une technique dénuée de toute fonction légitimante ». Un délégué explique d'ailleurs qu'il ne se « sent pas légitime en ce qu'aucun étudiant ne l'a légitimé à le représenter et à prendre des décisions pour lui »; un autre explique qu'il « est difficile de se sentir investi après être tiré au sort » (questionnaires envoyés aux délégués tirés au sort, extraits de réponse). Pour certains, on dépasse donc les sentiments d'adhésion procurés par l'inclusion communautaire ou la proximité, et l'individualisation semble parfois, poussée à l'excès, isoler les délégués sélectionnés par le sort. On peut faire l'hypothèse dans ce cas que la double composition de l'assemblée (23 tirés au sort et 23 membres d'associations facultaires) est une raison de ce décrochage, puisqu'elle rend visible dans la même assemblée un fonctionnement sérieusement différent, 
valorisant le petit groupe, l'association, la compagnie durant les assemblées et la discussion en groupe restreint.

Il faut dire enfin que cet effet d'effacement se double parfois d'un effet de désincarnation. En effet, certains délégués affichent une perte de confiance ou un certain désengagement. À la FAE, le tirage au sort ne semble pas être une source de richesse pour l'expression des personnalités mais plutôt une source d'insatisfaction ou de démotivation. Les effets égalitaires, communautaires et de neutralité ont un impact tel qu'ils peuvent déresponsabiliser le représentant ou en tout cas ne pas lui donner le poids nécessaire pour exprimer son avis face aux autres représentants lors de la délibération. Cette désincarnation peut poser deux défis au moins en matière de démocratie délibérative (Sintomer, 2011b). D'une part, «dans une perspective habermassienne, une bonne délibération est censée favoriser la force du meilleur argument [et pour ce faire], dans une société où les résistances structurelles des intérêts dominants sont énormes, les énergies suscitées par les passions semblent un combustible incontournable » (p. 178) que cette désincarnation paraît réduire. D'autre part, derrière les discours des représentants se cachent toujours des relations de pouvoir qui peuvent influencer la délibération et cette désincarnation peut « rester aveugle aux différences de capital social, économique ou politique des participants » (p. 177).

\section{Rapports de force internes et dynamiques de délibération}

La représentation, analysée jusqu'ici dans ses dimensions formaliste et symbolique, se réalise au final dans la délibération. Sans revenir sur les débats théoriques autour de la «démocratie délibérative», il faut considérer ici le moment de la délibération comme un lieu primordial pour comprendre la dialectique démocratique entre l'institution et les individus. Or, comme nous le rappelle Pierre Bourdieu (1979, p. 484), dans un contexte de domination, «l'opinion politique n'est pas un jugement pur et purement informatif capable de s'imposer par la force intrinsèque de sa vérité, mais une idée-force, enfermant une prétention d'autant plus grande à se réaliser [...], que le groupe qu'elle mobilise par son efficacité proprement symbolique est plus nombreux et plus puissant ». Il faudra se demander ici si le tirage au sort permet, en affaiblissant les structures, de faire glisser la conception de la délibération d'une opposition d'idées-forces vers la confrontation pacifiée d'idées pures ou si, au contraire, dans ce contexte, les dynamiques d'acteurs et de groupes ne permettent pas un tel changement.

\section{Compétence, participation et connaissance du jeu politique}

Selon Loïc Blondiaux (2008, p. 42), « L'idéal délibératif requiert en théorie pour les participants non seulement une égalité dans la prise de parole mais aussi 
dans la capacité à recourir à des arguments persuasifs, c'est-à-dire susceptibles d'être pris en compte dans la décision finale ». Il est difficile ici de juger la compétence politique du point de vue du capital scolaire puisque les délégués sont tous des étudiants. Il existe certes des différences au sein du corps estudiantin (Michon, 2008), mais face à la puissance du « filtre universitaire $»^{12}$, les données récoltées sur ce point ne permettent pas de dégager ici des tendances claires. L'analyse empirique comparative (entre les élus et les tirés au sort) oblige plutôt à considérer un autre type de capital, qui peut être perçu comme une variation du capital scolaire et culturel : la culture politique, entendu comme la compétence à participer au jeu politique, l'ignorance des codes spécifiques de ce jeu pouvant être à l'origine du sentiment d'incompétence qui pousse les profanes à la retenue. Dans cette optique, je construis trois types de compétences politiques en me référant aux travaux de Gil Delannoi (2011a).

D’abord, la compétence technique, c'est-à-dire la connaissance des normes et du langage politique, nécessaire à la participation active au débat. À la FAE, l'assemblée législative demeure un lieu dans lequel la délibération s'avère passablement codifiée. La participation y constitue un exercice spécifique, liée à la maîtrise d'un langage particulier («ordre du jour», «procès-verbal», « motion», «liste de parole», «question directe», etc.). La méconnaissance ou l'incompréhension de ces normes est à l'origine pour une majorité des tirés au sort d'une incompétence relative, ou plutôt du sentiment d'incompétence partagé par certains acteurs, qui tend à favoriser les membres possédant ces ressources institutionnelles, par leur fonction, leur ancienneté ou leur formation politique au sein d'une autre organisation.

À cette incompétence technique peut s'ajouter l'incapacité concrète face à la complexité de certains sujets, cristallisant la méconnaissance factuelle du sujet politique traité. C'est ce qu'on peut nommer la compétence substantielle. La politique de la formation, qui occupe l'assemblée de la FAE, est un domaine politique complexe, à cause du système fédéraliste suisse en la matière. Or, un des prérequis à l'élaboration d'une opinion éclairée est que les citoyens puissent débattre de façon informée (Habermas, 1996). Face à la complexité des sujets, les délégués tirés au sort s'avouent souvent démunis - à cause du manque de temps - pour comprendre les sujets traités, sans pour autant s'auto-exclure du débat par des justifications d'impuissance du type «ce n'est pas mon affaire » ou «ça ne m'intéresse pas ». Ils s'en remettent plutôt à la position de membres plus anciens, au bureau ou s'abstiennent. Cet effet va probablement de pair avec l'effet symbolique d'infériorité décrit plus haut.

[12] En Suisse, $23 \%$ des femmes et $28 \%$ des hommes ont effectué en 2014 des études dites supérieures, études relativement sélectives en comparaison européenne. OFS, 2015, Statistiques de l'éducation 2014, Neuchâtel, p. 3. 
Enfin, il ne faut pas négliger la compétence politique ou politicienne, c'est-à-dire la capacité de compréhension fine du jeu politique : savoir que dire, quand le dire, comment le dire, et comprendre qui détient le pouvoir. Dans ce cas, le tirage au sort ne semble pas engager un effet assez puissant pour déstructurer les normes et les codes classiques du jeu politique dans cette assemblée. La discussion avec un représentant des associations, membre de l'assemblée depuis plusieurs années, illustre bien ce point :

« Les deux premières $A D$, c'est mon suppléant ${ }^{13}$ qui est allé à ma place et il s'est fait entourlouper, il s'est fait prendre dans le débat, enfin... il s'est fait prendre par le débat [il souligne]. Et il n'a rien obtenu. Alors évidemment qu'il avait une position très à gauche et probablement une position très arrêtée, mais il s'est fait attraper par le débat qu'il ne connaissait pas » (entretien Émilien).

D’un point de vue général, les délégués tirés au sort semblent donc souffrir dans la délibération d'une triple incompétence qui les pousse à la délégation. À l'inverse, ce sont les membres qui ont du temps, de l'expérience (bureau) ou qui préparent les débats ensemble (associations facultaires) qui font vivre la délibération.

\section{Le rôle du bureau exécutif et l'encadrement de la délibération}

Le déséquilibre entre les profils des membres de l'assemblée législative et des autres organes de l'association a donc un impact sur la délibération. Dans la pratique, c'est en effet le bureau qui s'occupe au jour le jour des tâches politiques et des projets de l'association et qui est formellement désigné pour diriger l'assemblée et encadrer les débats, ce qui le conduit parfois à outrepasser son rôle et à prendre des décisions qui sont d'ordre législatif. L'analyse empirique des exemples délibératifs récents (Blondiaux, 2008) a montré l'importance, pour la qualité de la délibération, d'une animation dynamique et équilibrée des débats qui permettrait de neutraliser les différences de profil des participants. À la FAE, la gestion des débats reste orientée puisque le bureau est directement partie prenante. Il est fréquent d'observer la coprésidence, qui a la gestion de séance, faire des commentaires sur les interventions ou sur le déroulement de l'assemblée (par exemple: «Ah, on arrive à un sujet très intéressant »). La gestion du temps semble aussi passablement influencer la bonne tenue des discussions puisqu'elles sont souvent arrêtées rapidement par la gestion de séance. J'ai pu observer, par exemple, plusieurs délégués proposer un débat sur un sujet que le bureau a refusé d’organiser en expliquant « qu'en cas de long débat, l'assemblée se terminerait à minuit ».

Le déséquilibre n'est cependant pas dépendant des personnalités des membres du bureau, mais est bien structurel entre un bureau semi-permanent et les

[13] Il n'existe des suppléants que pour les représentants des associations facultaires, ce qui n'est pas le cas des élus puis des tirés au sort. 
membres de l'assemblée législative tirée au sort. Ce déséquilibre structurel, observé avec l'introduction de la sélection aléatoire, favorise la division du travail, la délégation et la passivité des membres de l'Assemblée des délégué.e.s au profit des membres du bureau exécutif. Au contraire, avant 2012, les représentants politisés constituaient un véritable contrepoids à l'exécutif, les groupes politiques ne craignant nullement de remettre en cause la parole de ces semiprofessionnels, et jouant ainsi un véritable rôle de contre-pouvoir que n'assument plus les délégués tirés au sort.

\section{Conflits, (dé)politisation et consensus d'illégitimité}

Sous l'influence de Jürgen Habermas enfin, les théoriciens politiques se sont interrogés sur les liens que certains dispositifs participatifs pouvaient établir entre participation et recherche de consensus (Blondiaux, 2008), notamment en opposant la délibération consensuelle ${ }^{14}$, plus apaisée et raisonnable, à la délibération conflictuelle. Avant l'introduction de la sélection aléatoire, le débat à la FAE était particulièrement conflictuel entre les listes partisanes. C'était d'ailleurs une des raisons de ce changement. Toutefois, après l'introduction du tirage au sort, la délibération n'est pas totalement devenue constructive et consensuelle. J'observe plutôt deux glissements. D'abord, un glissement - selon les concepts de Jane Mansbrige (2013) - d'une optique agrégative de la délibération vers une optique plus délibérative de celle-ci, sans pour autant atteindre dans ce cas cet idéal-type. Par la suppression des listes politisées, la sélection aléatoire semble ramener la formation de l'opinion au sein de l'assemblée : sans idée préconçue ou discutée préalablement en groupe, les délégués tirés au sort attendent souvent le moment du débat pour faire leur choix. D’autre part toutefois, la décision ne semble progressivement plus résulter à la FAE d'un vrai débat d'idées (peu importe qu'on considère celles-ci comme des idées pures ou des idées-forces), mais peut plutôt être considérée comme le résultat d'un consensus d'illégitimité, les délégués ne se sentant plus légitimes à avancer un point de vue et préférant s'abstenir :

«Ce n'est pas parce qu'on vote sur tout qu'il y a une bonne qualité des débats. Des fois, je me demande même si ce cadre-là n'est pas ce qui rend le débat stérile... [...] Si tu vois que personne ne vote oui, alors je vais me fondre dans la masse... Donc tu te dis, c'est très démocratique parce qu'on vote sur tout mais cette démocratie a des limites parce que (silence) c'est comme si c'était une unité qui pense tout pareil, alors que ce n'est pas le cas, on sait bien que c'est impossible. Cinquante personnes qui pensent tous la même chose, c'est impossible » (entretien Luc).

[14] Philippe Urfalino (2007) a développé le concept de « consensus apparent » pour montrer que l'idée de consensus n'est en fait souvent qu'une décision où ceux qui pourraient mettre leur veto s'abstiennent de le faire même s'ils ne sont pas convaincus. 
Mes observations et les entretiens menés m'ont rapidement confirmé ce deuxième glissement par lequel les représentants tirés au sort, de manière générale, s'engagent beaucoup moins dans la délibération que les représentants élus ou les membres des associations. Alors qu'avant l'instauration du tirage au sort, les élus monopolisaient la parole lors des débats au détriment des associations facultaires, ce sont dès lors majoritairement les membres des associations de faculté qui débattent avec les membres du bureau et très peu les délégués issus du tirage au sort qui, pour leur part, adoptent une posture bien plus passive. Ceux-ci sont tous assis seuls à une table, plutôt à l'avant de l'auditoire dans lequel se déroule l'assemblée, osent moins prendre la parole et discutent peu entre eux légalement pendant les pauses ou après l'assemblée). Lors des délibérations, ils ont certes une plus grande écoute de ce que disent les autres (peu sont sur les réseaux sociaux et écoutent les débats, par exemple), mais cette écoute se double d'une plus grande passivité lors des débats, qui se matérialise finalement par une augmentation de l'abstention lors des votes, comme si le système délibératif de l'association allait trop vite pour une prise de décision réfléchie et posée. À l'inverse, les membres des associations sont assis en groupe (par associations facultaires), et adoptent une posture bien plus relâchée lun membre pose ses pieds sur la table, un groupe boit des bières tout au long de l'assemblée et d'autres discutent ou s'écrivent via les réseaux sociaux). Un délégué tiré au sort l'exprime ainsi :

« Ce n'est pas évident de prendre la parole devant une quarantaine de personnes, souvent qui sont impliquées politiquement, qui connaissent le système, qui sont capables d'argumenter, qui sont capables de tenir un discours militant » (entretien Émilien).

Il existe donc une véritable inégalité structurelle entre les membres de l'assemblée, qui déploie des effets certains sur le déroulement concret de la délibération. On peut faire l'hypothèse que ce sentiment d'infériorité trouve ses origines dans deux causes principales. Premièrement, la structure du dispositif n'impose pas un turn-over des délégués des associations facultaires. De facto, certains délégués - auxquels s'ajoutent les membres du bureau - se retrouvent en position d'experts déjà au fait des mécanismes de discussion et des sujets traités par la FAE. Mais face à cette «interdiction » positionnelle et technique de la prise de parole s'ajoute, deuxièmement, la force du nombre, des groupes lassociations, bureau, et avant 2012 des listes politisées) auxquels ne peuvent plus se rattacher les représentants tirés au sort, isolés des structures investissant le locuteur d'une force collective qui favorise la prise de parole. C'est en quelque sorte une variation du capital social que de se retrouver membre d'une organisation et qui permet d'encourager la prise de rôle. Pour reprendre l'analyse de J. Mansbridge (2013, p. 64) on peut dire que le développement de groupes, par leur force socialisatrice, « encourage les individus à se percevoir avant tout comme des membres de (sous-communautés) et est susceptible d'éroder des liens censés unir entre eux » les individus d'une même communauté (Gerth, Wright Mills, 1954 ; Paugam, 2012). 
En ce sens, si la présence de listes politisées pouvait porter atteinte à l'intégrité d'un corps estudiantin plus large, n'étaient-elles pas constitutives, au contraire, de "l'ancrage expérientiel de la participation des individus à la collectivité » (Mansbridge, 2013, p. 64), notamment en discutant régulièrement et collectivement des différents sujets et en organisant des séances de préparation communes avant les assemblées ? Peut-être également que les élus, apprentis du champ politique, étaient pour certains mus par l'idée de "s'entraîner au débat » dans le cadre de la FAE. Dans tous les cas, si le tirage au sort permet d'apaiser et de rationaliser la délibération, il ôte ici symboliquement le droit à certains représentants d'avoir leur propre avis, comme s'il avait pour effet de dépolitiser plutôt que de démocratiser:

«Il n'y a pas de grandes discussions à la FAE. C'est vraiment discuté sur très peu. Il n'y a pas de tranchage. Tout le monde vote oui ; tout le monde vote non. [...] Il n'y a jamais eu, et c'est ce que je regrette d'ailleurs, un cas de figure où tu te sens investi par une pulsion parce qu'il y a réellement un débat. Non... le débat est creux, il est unilatéral » (entretien Luc).

Dans notre cas, la sélection aléatoire a donc presque totalement supprimé la possibilité d'une pluralité de points de vue et d'arguments, qui sont pourtant une condition essentielle pour faire émerger de l'information et arriver à un réel consensus d'idées (Manin, 1985, p. 82). Or, les décisions sont souvent prises à la FAE dans le même sens que les solutions proposées par le bureau, sans être le résultat d'une véritable discussion. Le tirage au sort a fortement modifié les dynamiques de délibération au sein de l'assemblée en neutralisant la logique agonistique du débat à un point tel qu'il a aussi en partie supprimé le caractère actif de la représentation.

\section{Le tirage au sort à la FAE : une procédure démocratique?}

La FAE constitue donc un environnement particulièrement pertinent pour mener une analyse des dynamiques démocratiques d'une assemblée délibérative. Bien que cet exemple puisse se revendiquer d'un contexte politico-idéologique commun à d'autres pratiques contemporaines réactivant le hasard, il ne faut pas oublier que le tirage au sort est neutre en tant que mécanisme de sélection (Delannoi, 2011b), et ne porte pas intrinsèquement cette idée démocratique et égalitaire : c'est son application concrète et la culture de l'institution dans laquelle il est appliqué qui lui attribuent ses valeurs.

Dans cette perspective, la technique de la sélection aléatoire semble bien dans notre cas «faire abstraction du poids fonctionnel des unités [qu'elle] traite comme substituables, anéantissant par là les structures, celles des champs 
par exemple » (Bourdieu, 2001, p. 9) ${ }^{15}$. C'est en effet la multiplication des profils, dans le sens d'un plus grand accès de profanes au lieu de l'assemblée, et par là même la constitution d'une assemblée plus représentative des étudiants, qui peut être observée par l'utilisation du tirage au sort à la FAE. La sélection aléatoire réduit en ceci la force des structures génératrices de rôles, et limite la carrière politique, le monopole des partis et la concentration des pouvoirs aux mains de quelques profils typiques. Toutefois, cette déstructuration déploie aussi des effets symboliques qui poussent à la désincarnation et à l'effacement des délégués dans la délibération. En ce sens, la déstructuration des groupes défendant des intérêts communs (les partis par exemple) peut être dangereuse, particulièrement pour les groupes dominés pour qui la nécessité de s'assembler pour défendre des intérêts communs est fondamentale.

De plus, le tirage au sort ne semble pas engager des effets assez puissants pour déstructurer les normes et les codes classiques du jeu politique au sein de cette association. Posséder les compétences « politiciennes » et «techniques » reste encore une condition nécessaire pour peser véritablement dans la décision finale au sein de l'assemblée législative de la FAE. La suppression des listes politisées, qui se double d'une disparition de représentants au fait de ces codes agissant comme un contre-pouvoir, permet alors un déplacement dans la délibération, du rapport de force vers le bureau exécutif.

Il est donc difficile de rapprocher cette expérience de celle des jurys, des assemblées citoyennes ou des conférences de consensus, dans lesquels les enquêtes sont unanimes pour dire que la qualité des débats ainsi que la formation d'une opinion plus éclairée en sont améliorées. Cet exemple montre qu'introduire la sélection aléatoire dans une structure qui rappelle les dispositifs du gouvernement représentatif ne permet de loin pas à elle seule de démocratiser les dispositifs représentatifs. La configuration propre à la FAE révèle une posture particulière des tirés au sort isolés - qui sont en quelque sorte des représentants-amateurs - au regard de la logique classique et codifiée de la représentation politique. En effet, la nécessité de la connaissance des codes propres au champ politique prend ici toujours en partie le dessus sur la compétence substantielle qui devrait être primordiale dans un débat démocratique, confirmant l'intuition de G. Delannoi (2011a, p. 135) que dans nos systèmes « l'idée de compétence technique [s'est] déjà substituée à l'idée de compétence démocratique ».

Néanmoins, le niveau de connaissance des codes et des normes politiques n'est pas définitivement figé et leur compréhension peut naître justement en les pratiquant. L'apprentissage de la participation politique demeure un des grands

[15] Si Pierre Bourdieu discute par cette formulation de la technique de l'échantillon aléatoire dans le cadre de l'enquête sociologique, on peut appliquer son analyse à la sélection aléatoire en politique, notamment au regard de ce qu'il en dit dans son texte sur la représentation politique (1981). 
apports de l'introduction du tirage au sort et de la rotation des charges dans ce cas. C'est la conception qu'un citoyen doit être capable de bien gouverner let bien être gouvernél et que cette capacité s'apprend dans l'alternance des rôles. C'est en soit l'apprentissage de la gouvernance. Le tirage au sort sert dans une certaine mesure à cet apprentissage. Un délégué nous explique par exemple que « les termes précis, genre ordre du jour, je connaissais sans forcément comprendre ce que c'était, mais à partir du moment où on y est c'est comme si... d'un coup on est éclairé (rires) ! Quand on n'est pas dedans ça reste abstrait. Mais une fois que tu te contrains vraiment, ça devient plus clair » (entretien Massimo). Il ne faut donc pas négliger à long terme les effets symboliques du tirage au sort qui semblent particulièrement puissants ${ }^{16}$.

\section{Bibliographie}

Bacqué M.-H., Sintomer Y. (dir.), 2010, La démocratie participative inachevée : genèse, adaptations et diffusions, Paris, Éditions Yves Michel.

Barber B., 1997 [1984], Démocratie forte, trad. J.-L. Piningre, Paris, Desclée de Brouwer.

Blondiaux L., 2001, « Démocratie locale et participation citoyenne : la promesse et le piège », Mouvements, 18, p. 44-51.

Blondiaux L., 2008, Le nouvel esprit de la démocratie. Actualité de la démocratie participative, Paris, Seuil.

Blondiaux L., Sintomer Y., 2002, « L’impératif délibératif », Politix, 57, p. 17-35.

Bourdieu P., 1979, La distinction. Critique sociale du jugement, Paris, Éditions de Minuit.

Bourdieu P., 1981, « La représentation politique. Éléments pour une théorie du champ politique », Actes de la recherche en sciences sociales, 36-37, p. 3-24.

Bourdieu P., 2001, « Le mystère du ministère. Des volontés particulières à la “volonté générale" », Actes de la recherche en sciences sociales, 140, p. 7-11.

Cervera-Marzal M., Dubigeon Y., 2013, « Démocratie radicale et tirage au sort. Au-delà du libéralisme », Raisons politiques, 50, p. 157-176.

Courant D., 2014, Tirage au sort et concertation dans l'Armée française : Le cas du Conseil Supérieur de la Fonction Militaire (1969-2014), mémoire de recherche de master 2, EHESS (Paris).

Courant D., 2018, « Penser le tirage au sort. Modes de sélection, cadres délibératifs et principes démocratiques », in A. Chollet, A. Fontaine (dir.), Expériences du tirage au sort en Suisse et en Europe : un état des lieux, Berne, Schriftenreihe der Bibliothek am Guisanplatz.

[16] Je remercie Alexandre Fontaine pour ses précieux commentaires. 
Courant D., 2019, «Délibération et tirage au sort au sein d'une institution permanente. Le Conseil supérieur de la fonction militaire (1968-2016)», Participations, 23, p. 61-91.

Dahl R. A., 1989, Democracyand Its Critics, New Haven/Londres, Yale University Press.

Delannoi G., 2011a, « Le tirage au sort, un et multiple », Esprit, 8-9, p. 134-135.

Delannoi G., 2011b, « Le tirage au sort: une approche démocratique », Esprit, 8-9, p. 153-161.

Fourniau J.-M., 2019, « La sélection des mini-publics entre tirage au sort, motivation et disponibilité », Participations, hors-série 2019 (Tirage au sort et démocratie. Histoire, instruments, théories), p. 373-400.

Garibay D., 2015, «Vingt-cinq ans après Porto Alegre, où en est (l'étude de) la démocratie participative en Amérique latine ? », Participations, 11, p. 7-52.

Gaxie D., 1993 [1978], Le Cens caché. Inégalités culturelles et ségrégation politique, Paris, Seuil.

Gaxie D., 1997, « Homogénéité et hétérogénéité du personnel politique », Aprèsdemain, 399, p. 28-32.

Gerth H., Wright Mills C., 1954, Character and Social Structure. The Psychology of Social Institutions, Londres, Routledge.

Göhler G., Pégny G., Sintomer Y., 2013, « La dimension affective de la démocratie. Réflexion sur la relation de la délibération et de la symbolicité », Raisons politiques, 50, p. 97-114.

Habermas J., 1996, Droit et démocratie. Entre faits et normes, Paris, Gallimard.

Hayat S., 2013, « La représentation inclusive », Raisons politiques, 50, p. 115-135.

Hayat S., Sintomer Y., 2013, «Repenser la représentation politique », Raisons politiques, 50, p. 5-11.

Lagroye J. (dir.), 2003, La politisation, Paris, Belin.

Landemore H., 2010, «La raison démocratique : les mécanismes de l'intelligence collective en politique », Raison publique, 12, p. 9-56.

Le Bart C., Merle P., 1997, La citoyenneté étudiante. Intégration, participation, mobilisation, Paris, Presses universitaires de France.

Le Mazier J., Testi J., Vila R., 2014, « Les voies multiples de la représentation en situation de délégation ratée : agir au nom des étudiants », in A. Mazeaud (dir.), Pratiques de la représentation politique, Rennes, Presses universitaires de Rennes, p. 203-227.

Malkopoulou A., 2014, « The paradox of democratic selection: is sortition better than voting? », in K. Palonen, R. María (dir.), Parliamentarism and democracy theory historical and contemporary perspectives, Opladen/Toronto, Barbara Budrich, p. 229-253.

Manin B., 1985, « Volonté générale ou délibération. Esquisse d'une théorie de la délibération politique », Le Débat, 33, p. 72-93. 
Manin B., 2012 [1995], Principes du gouvernement représentatif, Paris, Flammarion.

Mansbridge J., 2013, « Les Noirs doivent-ils être représentés par des Noirs et les femmes par des femmes ? Un oui mesuré », Raisons politiques, 50, p. 53-78.

Mellina M., 2016, Démocratiser la démocratie ? Le tirage au sort de l'Assemblée des délégué.e.s de la Fédération des associations d'étudiant.e.s de l'UNIL, mémoire de recherche de master, Université de Lausanne.

Michon S., 2008, « Les effets des contextes d'études sur la politisation », Revue française de pédagogie, 163, p. 63-75.

Paugam S., 2012, « “Compter sur" et "compter pour”. Les deux faces complémentaires du lien social », in R. Castel, C. Martin (dir.), Changements et pensées du changement. Échanges avec Robert Castel, Paris, La Découverte, p. 215-230.

Pitkin H. F., 1972 [1967], The concept of representation, Berkeley/Los Angeles, University of California Press.

Rancière J., 2005, La haine de la démocratie, Paris, La Fabrique.

Röcke A., 2006, « Représentation “miroir" et démocratie : le tirage au sort dans les jurys citoyens berlinois », Politique et Sociétés, 25 (1), p. 13-30.

Rosanvallon P., 2008, La légitimité démocratique: impartialité, réflexivité, proximité, Paris, Seuil.

Ségur P., 2013, « Désignation des gouvernants par le tirage au sort », Revue française de droit constitutionnel, 95, p. 687-698.

Sintomer Y., 2011a, Petite histoire de l'expérimentation démocratique : tirage au sort et politique d'Athènes à nos jours, Paris, La Découverte.

Sintomer Y., 2011b, « Tirage au sort et politique : de l'autogouvernement républicain à la démocratie délibérative », Raisons politiques, 42, p. 159-186.

Sintomer Y., 2019, « De la démocratie délibérative à la démocratie radicale? Tirage au sort et politique au $x x^{e}$ siècle », Participations, 23, p. 33-59.

Stone P., 2009, « The logic of Random Selection », Political Theory, 37 (3), p. 375-397.

Suiter J., Farrell D., Harris C., O’Malley, 2019, « La première Convention constitutionnelle irlandaise (2013-2014) : un dispositif délibératif à forte légitimité ?», Participations, 23, p. 123-146.

Talpin J., 2019, « Le tirage au sort démocratise-t-il la démocratie ? Ou comment la démocratie délibérative a dépolitisé une proposition radicale », Participations, hors-série 2019 (Tirage au sort et démocratie. Histoire, instruments, théories), p. 453-473.

Urfalino P., 2007, « La décision par consensus apparent. Nature et propriétés », Revue européenne des sciences sociales, XLV (136), p. 47-70. 


\section{Abstract-Sortition in students' associations: A democratic experience at the University of Lausanne?}

In this article, we describe the use of sortition within the Fédération des associations d'étudiant.e.s, the student association of the University of Lausanne. In 2013, the association introduced sortition as a method to designate the representatives of its legislative assembly. Prior to this introduction, all students registered at the University of Lausanne voted to designate their representatives during the academic year. This contribution analyzes the political dynamics of this particular experience in order to bring new elements and arguments to the field of democratic representation. It shows that this method does indeed diversify the profiles of representatives in order to enhance their representativeness. It also shows that sortition also has symbolic effects, which lead to a greater consideration of the community's interest in the deliberation. Finally, it discusses how these representative and symbolic dimensions are realized in the dynamic process of deliberation. On this basis, this article concludes that sortition reduces the strength of role-generating structures, but not enough to completely dismantle the classic norms and codes of the political game.

Keywords Sortition, Random selection, Democracy, Representation, Deliberative democracy, Participatory democracy, Student association

Maxime Mellina est doctorant à l'Institut d'études politiques de l'Université de Lausanne et du département de science politique de l'Université de Paris 8. Il est membre du projet du Fonds national suisse de la recherche scientifique intitulé « Expériences de tirage au sort en Suisse ». Ses recherches portent sur la théorie de la démocratie et l'analyse sociohistorique des dispositifs de tirage au sort. Dans ce cadre, une attention particulière est portée à l'examen de la disparition du tirage au sort et de l'apparition du gouvernement représentatif à l'aube des révolutions françaises et américaines.

\section{Mots clés}

Tirage au sort, démocratie, représentation, démocratie délibérative, démocratie participative, associations étudiantes 